\title{
Influence of heritability on occlusal traits: a systematic review of studies in twins
}

\author{
Lucas Garcia Santana ${ }^{1 *} \mathbb{D}$, Carlos Flores-Mir ${ }^{2}$, Alejandro Iglesias-Linares ${ }^{3}$, Matheus Melo Pithon ${ }^{4}$ and \\ Leandro Silva Marques'
}

\begin{abstract}
Background: The aim of this systematic review was to identify, evaluate, and provide a current literature about the influence of heritability on the determination of occlusal traits.

Materials and methods: MEDLINE, SCOPUS, Web of Science, LILACS, and Google Scholar were searched without restrictions up to March 2020. Studies with twin method were considered and the risk of bias assessment was performed using quality of genetic association studies checklist (Q-Genie). The coefficient of heritability $\left(h^{2}\right)$, modelfitting approaches, and coefficient correlation were used to estimate the genetic/environmental influence on occlusal traits. The GRADE tool was used to assess the quality of the evidence.

Results: Ten studies met the eligibility criteria. Three studies presented good quality, five moderate quality, and two poor quality. Most studies have found that the intra-arch traits, mainly the maxillary arch morphology, such as width ( $\left.h^{2} 16-100 \%\right)$, length ( $\left.h^{2} 42-100 \%\right)$, and shape ( $\left.h^{2} 42-90 \%\right)$, and the crowding, mainly for mandibular arch $\left(h^{2} 35-81 \%\right)$, are under potential heritability influence. The traits concerning the inter-arch relationship, as overjet, overbite, posterior crossbite, and sagittal molar relation, seem not to be genetically determined. The certainty of the evidence was graded as low for all outcomes.

Conclusions: Although weak, the available evidence show that the heritability factors are determinant for the intraarch traits, namely, arch morphology and crowding. Possibly due they are functionally related, the occlusal traits concerning the maxillary and mandibular relationship seem to have environmental factors as determinants. In this scenario, early preventive approaches can offer a more effective and efficient orthodontic treatment.
\end{abstract}

Keywords: Systematic review, Twin study, Heritability, Dental variation

\section{Introduction}

Heritability is commonly defined as the total of phenotypic differences explained by genetic influence [1]. Dominance/recessive relationships within alleles that modulate the trait can have an effect on the character of the variation observed, that is, an allele within a gene with a dominant effect may alter the distribution or cause a discontinuous characteristic [1-3].

The genetic basis of dentoalveolar development has attracted considerable interest in orthodontics and

\footnotetext{
* Correspondence: lucasgarciasantana@gmail.com

${ }^{1}$ Department of Pediatric Dentistry and Orthodontics, Universidade Federal

dos Vales do Jequitinhonha e Mucuri, Diamantina, Minas Gerais, Brazil

Full list of author information is available at the end of the article
}

dentofacial orthopedics $[1,4,5]$. Previous studies assessing occlusal traits, such as maxillary and mandibular arch length and width, have provided some estimates of relative genetic and environmental influences [6-9]. Although genetic variance can be discerned for different occlusal variables, heritability tended to be low, emphasizing the importance of environmental influences on occlusal variation among siblings [6-9]. On the contrary, other studies have suggested that heredity plays a significant role in determining the morphology of the dentoalveolar arch, crowding amount, tooth spacing, and overbite degree $[5,10,11]$.

In this context, studies with a twin model, when appropriately applied, is one of the most effective 
instrument in assessing the relative contribution of genetic and environment factors to relevant occlusal traits [5]. When there is a high correlation for a certain trait in pairs of monozygotic (MZ) or dizygotic (DZ) twins, it is strongly suggested that genetics is the primary etiology $[12,13]$. Thus, twin studies make it possible to control potentially confounding variables related to genetic load, allowing, for example, to diagnose the actual contribution or influence of determinant factors for the etiology of malocclusions $[1,2,5]$.

A proper knowledge of the influence of heritability and the environmental factors on occlusal traits might increase our understanding of the etiology of malocclusions and therefore also of the limitations of orthodontic treatment. To the best of our knowledge, no systematic review has been carried out on this topic, and there is still considerable debate due to the lack of conclusive evidence. Based on these concepts, the aim of this systematic review was to identify the genetic influence in the establishment of malocclusions specifically considering studies in identical and fraternal twin individuals.

\section{Material and methods}

\section{Protocol and registration}

The report of this systematic review followed the guidelines of the Preferred Reporting Items for Systematic Review and Meta-Analysis (PRISMA) statement [14]. The study protocol was set a priori and registered on PROSPERO in August 2019 (CRD42019138059).

\section{Eligibility criteria}

For this systematic review, a PECOS question was established:

Population (P): MZ or DZ twin pairs with permanent dentition without previous or current orthodontic or orthopedic intervention;

Exposition (E): heredity background influence on occlusal traits in one of the twins;

Comparison (C): heredity background influence on occlusal traits in the other twins;

Outcomes $(\mathrm{O})$ : occlusal and dentoalveolar traits (overjet, overbite, arch morphology, crowding/spacing of teeth, crossbite, and sagittal relationship) quantified/ qualified through digital/plaster cast models, intra-oral measurements, or cephalogram;

Study design (S): observational twin studies such as cross-sectional, case-control studies, or data available at start from cohorts, and prospective studies with available diagnostic data at pre-treatment stage.

\section{Exclusion criteria}

The exclusion criteria were letters to the editor, editorials, case reports, case series, review articles, abstracts and discussions, sample with patients in deciduous or mixed dentition at the time of the analysis, individuals with tooth extraction or any other surgical procedure, and studies evaluating individuals with craniofacial deformities, syndromes, and cleft lip palates.

\section{Information sources and search strategy}

Electronic searches in MEDLINE (PubMed), Web of Science, SCOPUS, and LILACS, with no publication date restrictions, were conducted up to March 2020. The search strategy was originally planned for PubMed and subsequently adapted for the other databases (Appendix). Manual searches on the references of the included articles and search in the Google Scholar were also carried out to assess partially the grey literature. There was no restriction of language for inclusion.

\section{Study selection}

The selection of the studies consisted of two phases. During the first phase, two authors (LGS, LSM) independently examined the titles/abstracts. Those references that met the eligibility criteria were included. Full-text of references with insufficient information in the title/abstract for a final decision on inclusion or exclusion was retrieved for evaluation. The studies that appeared to meet the inclusion criteria were selected for full-text analyses. The inclusion and exclusion criteria were applied independently by the same authors, and those studies that met the eligibility criteria were included. In both phases, divergences were resolved by consensus.

\section{Data extraction and items extracted}

The data extraction of the included articles was performed independently and in duplicate by two authors. A standardized table was used to extract the data. The following data were extracted: author and year of publication, country (ethnic origin of the sample), study design, sample, age, and variables (occlusal and dentoalveolar parameters) in addition to the analysis of results. Data was compared for accuracy, and any discrepancy was resolved through the reexamination of the original study.

\section{Assessment of bias risk within studies}

As described elsewhere [15], quality, internal validity, and risk of bias of the included studies were assessed using the validated quality of genetic association studies checklist (Q-Genie) [15]. The Q-genie tool consists of 11 questions, which address the following aspects of study methodologies: study rationale, outcome, comparability, exposure, bias, sample size, analyses, statistical methods and control for confounding, inferences for genetic analyses, and inferences drawn from results. Each question has 7 possible answers as follows: "1 (poor)," "2," "3 (good)," "4," "5 (very good)," "6," "7 (excellent)." The overall quality of studies is classified as "poor quality" if 
score is $<35$, a score of 36-45 indicates "moderate quality" and a score of $>45$ indicates "good quality."

\section{Evaluation of the level evidence (risk of bias across studies)}

The level of evidence was assessed using the Grading of Recommendations, Assessment, Development and Evaluation Pro software (GRADEpro Guideline Development Tool, available online at gradepro.org) [16]. An adaptation to the realities of genetic association studies was needed. We planned to assess the certainty of the evidence on intra-arch (arch width and length, and tooth alignment) and inter-arch (overjet, overbite, sagittal molar relationship, and crossbite) outcomes. For each outcome examined, the GRADE assesses number of studies included, study design, risk of bias, inconsistency, indirectness, imprecision, and other considerations (such as publication bias). Depending on the seriousness of the limitation in each one of these domains, the evidence could be downgraded by one or two levels. Based on this assessment, the certainty of the evaluation of the outcome could be very low, low, moderate, or high quality.

\section{Summary measurements}

The coefficient of heritability $\left(h^{2}\right)$, model-fitting approaches, and coefficient correlation were used based on continuous or dichotomous data to estimate the genetic/ environmental influence on occlusal traits. The $h^{2}$ coefficient ranges from 0 to 1 (0 to 100\%). In general, the low magnitude (less than or equal to 0.20 ) can be explained when the effect of environmental factors is important and the correlation between genotype and phenotype is small. The moderate heritability coefficient ranges from 0.20 to 0.40 , and high when it is greater than or equal to 0.40 , in which case the correlation between the phenotype and the genotype of the patient is remarkable [17, 18]. The model-fitting method might be used to estimate the influence of additive genetic factors (A), nonadditive genetic factors (D), common or shared environmental factors $(\mathrm{C})$, and individual-unique environmental factors, including measurement error (E).

\section{Results}

\section{Study selection}

A complete search and selection flowchart is provided in Fig. 1. The search strategy yielded a total of 1319 references from the electronic databases. After the removal of duplicates and application of the eligibility criteria, 69 references were considered for full-text evaluation. The reasons for excluding studies eligible for full-text evaluation are given in the chart. At the end of the final eligibility evaluation phase, only 10 studies were included in this systematic review.

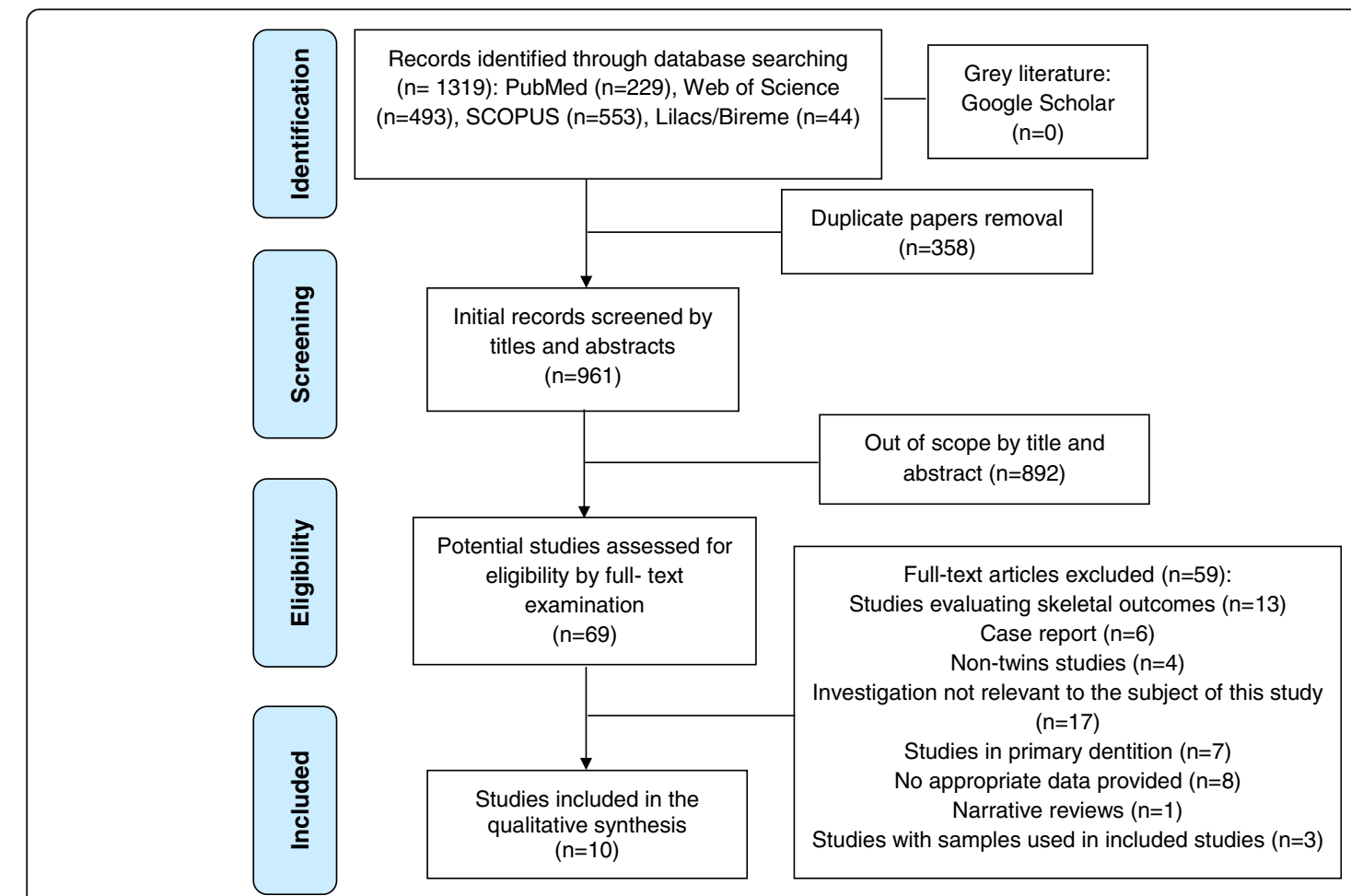

Fig. 1 PRISMA flowchart of article retrieval 


\section{Study characteristics}

Table 1 provides the descriptive characteristics of the 10 studies included in this systematic review. The studies were published between 1980 [17] and 2017 [19]. The studies included in this systematic review were conducted in six different countries, with most of the studies $(n=3)$ coming from the USA. All included studies were cross-sectional. The mean age of participants ranged from 14 [24] to 42 [22] years. One study [13] did not report the age of the participants; however, in all studies, the sample was evaluated in permanent dentition.

A total sample of 497 pairs of MZ twins and 366 pairs of DZ twins were evaluated. Nine studies evaluated both MZ and DZ twins, and only 1 study [19] evaluated exclusively MZ twins. Zygosity between twin pairs was confirmed by DNA testing in three included studies [7, $10,20]$. In four studies [13, 17, 23, 24], it was determined by serological for genetic markers, while one study [22] assigned using serologic and morphologic criteria together with dermatoglyphics. Two studies $[19,21]$ did not mention how zygosity between twins was confirmed.

Six studies used plaster dental casts [10, 17, 21-24] and one study used three-dimensionally scanned dental casts [7] to evaluate occlusal traits, such as overjet, overbite, posterior crossbite, arch morphology (shape, width, length, and asymmetry), molar sagittal position, tooth spacing, and crowding. One study [20] used cephalometric radiographs to evaluate overjet and overbite. One study [19] used the Peer Assessment Rating (PAR) index to evaluate occlusal parameters. Kawala et al. [13] did not report the survey method used for data collection.

The most common method (used in six studies [7, 13, $17,21,23,24])$ to estimate the strength of genetic and environmental contributions was through the $h^{2}$ coefficient. One study [20] used a model-fitting approach and one study [7] used both methods. Only two studies [19, 22] used the correlation coefficient as the main analysis.

\section{Risk of bias within studies}

The methodological appraisal of the included studies is reported in Table 2.

Studies were scored between 33 and 49 in the QGenie checklist. Three studies [10, 20, 22] were rated to have good quality, five studies as moderate quality [7, $17,21,23,24]$, and two studies [13, 19] rated to have poor quality. On average, included studies were rated as good for most of the items on the tool except for the domain "sample size and power" as studies had not described or determined the sample size required for their studies (convenience sample). Other items with the lowest classification were the "other sources of bias" and "selection and comparability of comparison group," due no information was provided regarding blinding of outcome assessment, and one study [16] evaluated a sample of twins born or residing in different countries, which may make the sample more representative.

\section{Synthesis of results}

Due to a lack of methodological, clinical, and statistical heterogeneity, a meta-analysis was not justifiable. Identified sources of heterogeneity were distinct methods for assessing the genetic contribution for occlusal parameters, different landmark references identified to evaluate the outcome, and heterogeneity between MZ and DZ twin samples.

\section{Results of individual studies}

Main findings of the included studies can be found in Table 1. The most occlusal traits evaluated were overjet and overbite (six studies evaluated both outcomes [13, 17, $20,22-24]$ ), and heritability estimates were generally low. Only one study [23] reported potential genetic dominance for overjet $\left(h^{2}=0.77, p=0.0001\right)$, while two studies reported for overbite [20, 24]. A model-fitting method has shown that overbite seems to be mostly determined by dominant genetic factors (74\%), less influenced by specific environmental factors represent (26\%) [20].

Genetic variation has a major effect on arch width and length. Four studies [7, 10, 17, 22] (out of a total of five studies $[7,10,17,22,23])$ found modest to high heritability potential on maxillary $\left(h^{2}\right.$ ranged from 0.16 [17] to 1 [10]) and mandibular ( $h^{2}$ ranged from 0.22 [17] to 1 [10]) arch width, being the traits most cited by studies with genetic dominance. This was confirmed in MZ and DZ twins reared apart [22]. One study [7] reported a greater environmental component (51\%) for the determination of mandibular second premolars width, while the distance between maxillary and mandibular lateral incisors, canines, molars, and maxillary interpremolares showed a high heritability estimates. Three studies [7, 10, 23] (out of a total of four studies [7, 10, 17, 23]) reported a relevant heritability coefficient for arch length. In common, in these studies, the highest heritability coefficients were for the maxillary arch $\left(h^{2}\right.$ range from 0.42 [17] to 1 [10]) than for the mandibular arch $\left(h^{2}\right.$ range from 0.28 [17] to 0.86 [7]).

Two studies $[17,21]$ evaluated the shape of the maxillary and mandibular arch and the presence of asymmetries. A genetic contribution to the arch shape was reported with the maxillary arch $\left(h^{2}=0.90\right.$ and $h^{2}=$ 0.42 for quartic and quadratic arch terms, respectively) being superior to the mandibular arch $\left(h^{2}=0.35\right.$ and $h^{2}$ $=0$ for quartic and quadratic arch terms, respectively) [21]. However, there was no pattern in the correlation values between the quadrants of the maxillary and mandibular arch, suggesting that, although opposing arches within the individuals are functionally related, they will 


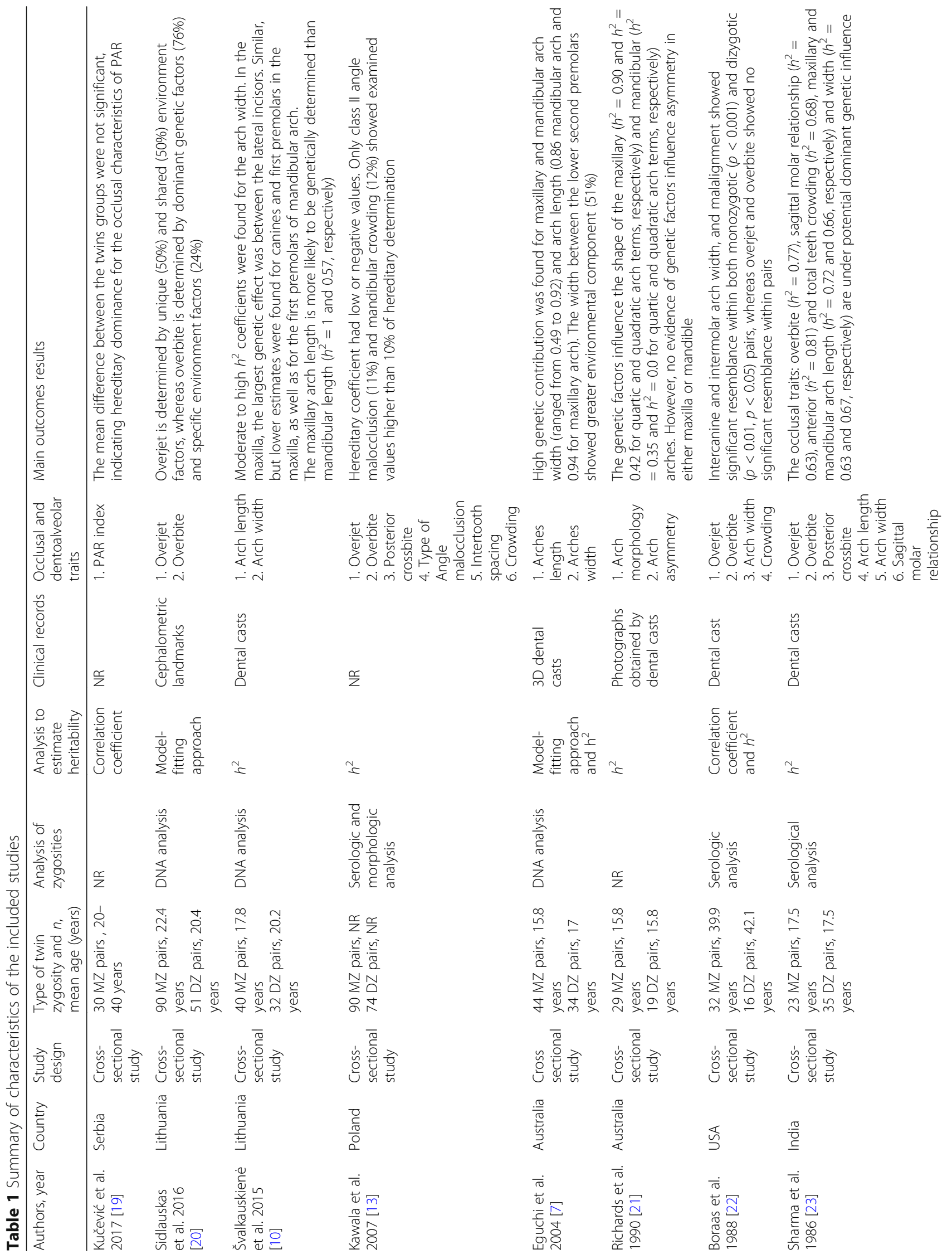




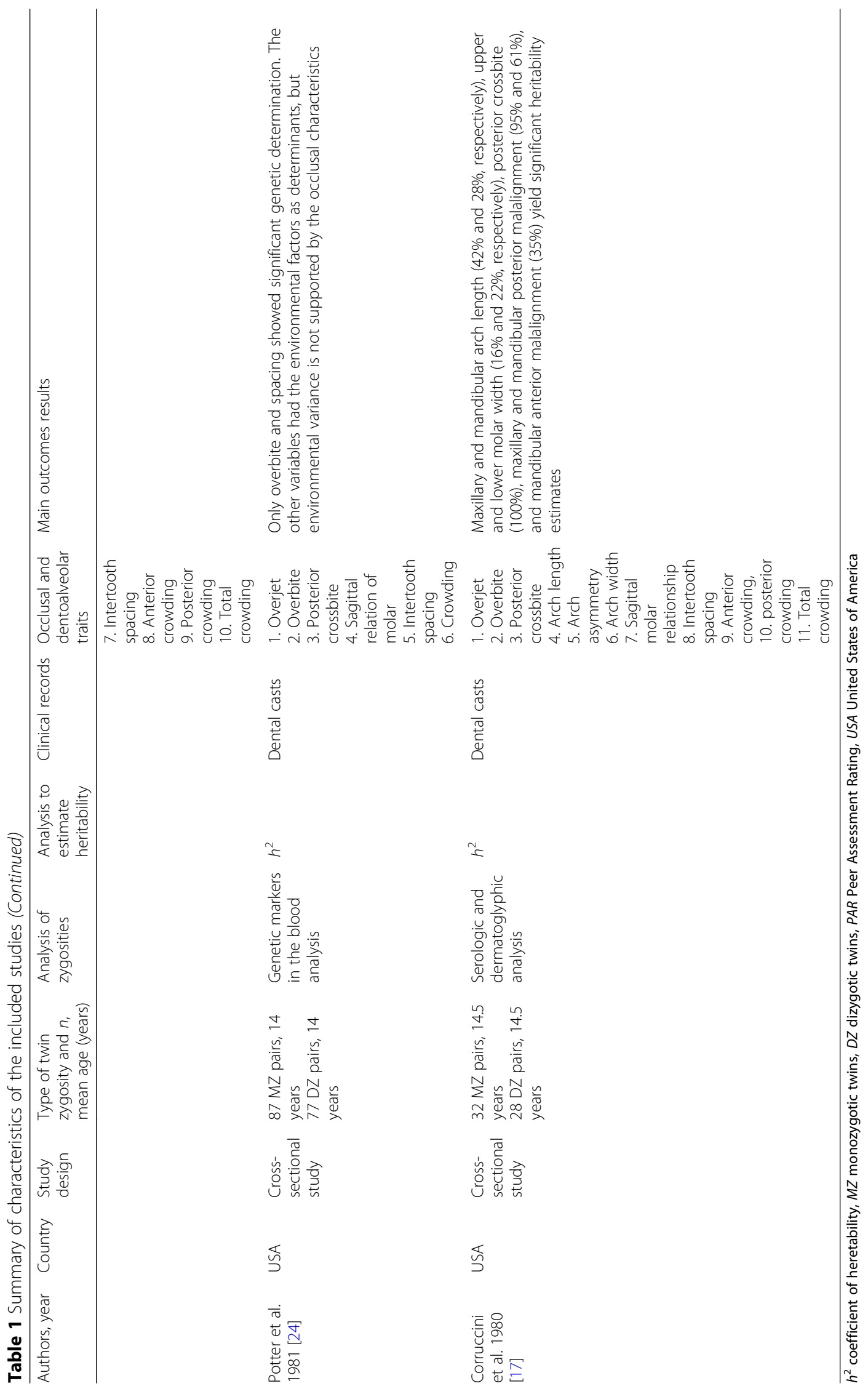


Table 2. Quality assessment scores for the selected studies based on the quality of genetic association studies (Q-Genie)

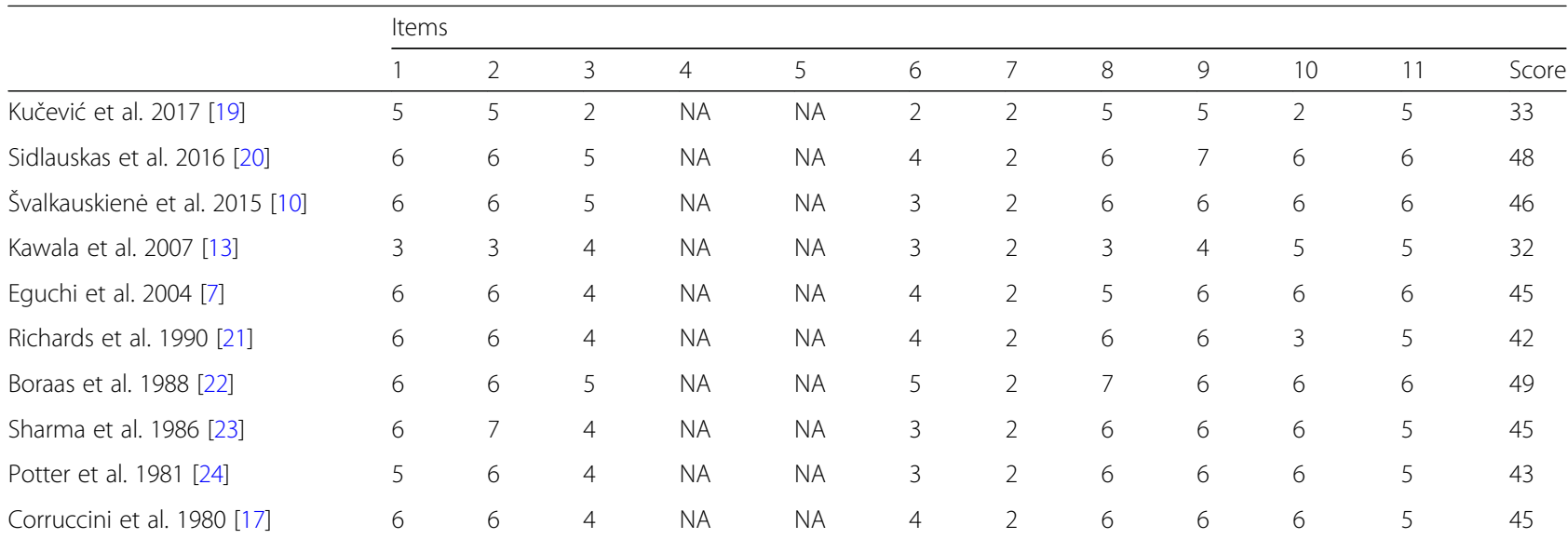

1 rationale for study, 2 selection and definition of outcome of interest, 3 selection and comparability of comparison group (if applicable), 4 technical classification of the exposure, 5 non-technical classification of the exposure, 6 other sources of bias, 7 sample size and power, 8 a priori planning of analysis, 9 statistical methods and control for confounding, 10 testing of assumptions and inferences for genetic analysis, 11 appropriateness of inferences drawn from results. All items have a maximum score of 7. NA not applicable

Table 3 GRADE evidence profile table about influence of heritability in main occlusal traits

\begin{tabular}{|c|c|c|c|c|c|c|c|c|}
\hline \multicolumn{9}{|c|}{ Certainty Assessment } \\
\hline $\begin{array}{l}\text { No. of } \\
\text { Studies }\end{array}$ & $\begin{array}{l}\text { Study } \\
\text { design }\end{array}$ & $\begin{array}{l}\text { Risk of } \\
\text { bias }\end{array}$ & Inconsistency & Indirectness & Imprecision & $\begin{array}{l}\text { Other } \\
\text { consideration }\end{array}$ & Impact & $\begin{array}{l}\text { Overall } \\
\text { certainty of } \\
\text { evidence }\end{array}$ \\
\hline \multicolumn{9}{|c|}{ Arch width } \\
\hline 6 & $O S^{a}$ & Serious $^{b}$ & Not serious & Not serious & Not serious & None & $\begin{array}{l}\text { Arch width has an important heritability } \\
\text { component }\end{array}$ & $\begin{array}{l}\oplus \oplus \circ \bigcirc \\
\text { LoW }\end{array}$ \\
\hline
\end{tabular}

Arch length

5 OS $^{a} \quad$ Serious $^{b}$ Not serious Not serious Not serious None

Overjet

6 OS $^{\mathrm{a}} \quad$ Serious $^{\mathrm{b}}$ Not serious Not serious Not serious None

Overbite

6 OS $^{\mathrm{a}} \quad$ Serious $^{\mathrm{b}}$ Not serious Not serious Not serious None

Malalignment/intertooth space

5 OS $^{\mathrm{a}} \quad$ Serious $^{\mathrm{b}}$ Not serious Not serious Not serious None

Sagittal molar relationship

4 OS $^{\mathrm{a}} \quad$ Serious $^{\mathrm{b}}$ Not serious Not serious Not serious None

Posterior crossbite

4 OS $^{\mathrm{a}} \quad$ Serious $^{\mathrm{b}}$ Not serious Not serious Not serious None component

Low

Genetic determination can be demonstrated with a $\oplus \oplus \circ$ higher heritability coefficient for the maxillary arch Low than for the mandibular

The heritability estimate for was not often $\bigoplus \oplus \bigcirc \bigcirc$ mentioned, highlighting a strong environmental contribution to observed variation

Low

Heritability was weak for overbite, suggesting the presence of hidden environmental determinacies

$\oplus \oplus \bigcirc \bigcirc$

LoW

The alignment of teeth, mainly in the mandibular $\oplus \bigoplus \bigcirc$ arch, is under strong dominance of genetic factors LoW

It appears that the environmental component as the major determinant

$\oplus \oplus \bigcirc$

Low

Although one study reports a high heritability $\quad \oplus \oplus \circ$ value, most studies did not shown a genetic Low 
not necessarily be similar in shape $(p>0.05)$ [21]. Thus, environmental influences are more determinant for the asymmetry of the maxillary and mandibular arches $\left(h^{2}=\right.$ $0, p>0.05)$ [17]. In the same way, three studies [13, 23, 24] (out of four studies [13, 17, 23, 24]) did not report that the posterior crossbite may be influenced by heritability factors.

Regarding the alignment/tooth spacing ratio, two studies $[17,23]$ reported that malalignment of the mandibular anterior teeth have significant heritability estimates ( $h^{2}$ range from 0.35 [17] to 0.81 [23]), while for maxillary arch no significant value were found $\left(h^{2}=0\right)$. Similarly, a significant $12 \%$ correlation was reported for hereditary determination of mandibular incisor crowding [13]. As far as posterior tooth rotation or displacement are concerned, one study [17] found a genetic dominance for the mandibular $\left(h^{2}=0.61, p=0.04\right)$ and maxillary $\left(h^{2}=\right.$ $0.95, p=0.0001)$ arches, while one study [23] reported no hereditary influence for this variable $\left(h^{2}=0, p=\right.$ 0.68 ). The presence of intra-arch spacing showed also modest heritability component [23, 24]. Finally, one study [22] found that tooth misalignment showed statistical significant differences in both MZ $(p<0.001)$ and $\mathrm{DZ}(p<0.01)$ twin pairs.

The role of heritability of the sagittal relationship examined at the level of first molars was evaluated in four studies [13, 17, 23, 24]. Three studies [13, 17, 24] concluded no or low influence of heredity $\left(h^{2}\right.$ range from 0.09 [17] to 0.11 [13]), while only one study [23] reported a significant influence of heredity for this parameter between pairs of twins $\left(h^{2}=0.63, p=0.006\right)$.

The only study [18] that used the PAR index did not report significant mean difference between twin groups, indicating hereditary dominance for the final PAR index value.

\section{Assessment of the certainty of evidence}

The certainty of evidence was evaluated according to the GRADE approach. The evaluated outcomes and reasons for downgrading the level of evidence are detailed in Table 3. The certainty level of evidence regarding the heritability of occlusal traits assessed was graded as low.

\section{External validity of findings}

The 10 studies included had convenience samples, as the twin sample was recruited from hospitals or universities. Due to the relative low prevalence of twins [25], it may be impractical to do a population study. However, it is noteworthy that studies included participants from various countries. This is important as the results were not restricted to a single geographic location or individuals exposed to the same local environmental factors, and possibly different ethnic origins were assessed.

\section{Discussion}

\section{Summary of evidence}

This systematic review raises the question to which extent occlusal traits are attributable to heritability of untreated orthodontically individuals in permanent dentition. For this purpose, heritability evaluation might be used as an initial approach in genetic studies, as it provides an estimate of how much phenotypic difference is conditioned by genetic influence $[18,20]$ Methodologies with twin studies provide them with the best information on the role of genetics versus environment in determining occlusal characteristics [2]. This is useful because the success of most orthodontic treatments depend on knowing the etiology of malocclusion for a problem-oriented approach [26].

Considering the fact that the GRADE tool suggested an overall low level of certainty, the results of this systematic review that included studies conducted on samples of twins of different nationalities suggests variable and frequently irrelevant heritability influence for overbite, overjet, sagittal molar relationship, and posterior crossbite, with highly accurate statistical analysis. Genetic variation seems to have an important effect mainly on width, length, and shape of the dental arch and the alignment/tooth spacing ratio, which were the most commonly reported occlusal characteristics in studies with genetically deterministic and/or higher heritability coefficients.

Genetic determination estimates for intra-arch traits that included teeth malalignments, teeth rotations, and inter-tooth spacing were significantly higher than the sagittal and vertical parameters evaluated. This is possibly related to the fact that certain occlusal traits such as overjet, overbite, and sagittal relationship of molars are more related to facial growth patterns, which, although genetically influenced, respond more to environmental variables such as long-term mouth breathing, allergic rhinitis, and minimized masticatory stress in fashions [5, $26,27]$. The similarity between the malposition's of the teeth between twins may well be due to the almost identical craniofacial form, which is more genetically conditioned [5].

Among the occlusal traits evaluated, the morphology of the dental arch appears to be the most genetically determined. Morphometric studies indicate greater heritability of body length measurements than for width measurements [28]. However, in addition to genetic influence for length, our results support substantial genetic component also for dental arch width and shape, although surprisingly, results point to a lack of genetic determination for posterior crossbite. According to the concept of balance between internal and external functional matrices, morphology and soft tissue behavior have a genetic component and significantly modifies 
dentoalveolar shape [26, 29]. Nevertheless, environmental factors such as deleterious oral habits, atypical swallowing, and mouth breathing may alter this internal matrix balance, leading to malocclusion [30, 31].

The low correlation between the shape of the maxillary and mandibular dental arch indicates that, although they are functionally related, the asymmetry in one arch does not need to be reflected by the asymmetry in the opposite arch. Possible differences in shape and symmetry between the opposite dental arches seem to be accommodated by differences in overjet or by the development of crossbite relationships.

Genetic influence was described to be weakly related with sagittal parameters, such as molar relationship, type of angle malocclusion, and overjet. This is surprising, as previous cephalometric studies have reported that the sagittal shape and position of the mandible are under genetic control in class II and class III relationships [20]. However, according to the reported scientific evidence, the heritability of these sagittal cephalometric parameters might not be under the same type of heritability pattern when referred to the patients' sagittal occlusion. A number of environmental variables have also been described as modifiers of jaws position. To this respect, hypertrophic tonsils, nasal blockage, birth anatomic defects, hormonal misbalances, non-physiological posture of tongue and soft tissues, and trauma/disease including premature loss of the first permanent molars $[5,29,32]$. The truth seems to lie in the interaction between genetic factors and environmental variables in the result of facial morphology and occlusal traits. Similarly, cephalometric studies reported higher heritability estimates for many vertical linear radiometric variables [33, 34]. However, our findings do not suggest this, as overbite, although reported as genetically determined by two studies, seems to be more related to sub-diagnosed environmental factors.

The correlation coefficient approach used to estimate heritability only compares the degree of association for selected traits between pairs of related individuals [2]. Thus, this method may not be the best estimate of heritability and, therefore, the results obtained in studies that used correlation coefficient should be interpreted with caution. The $h^{2}$ analysis provides a quantified estimate of the extent of genetic determination for phenotypic variation of the trait under investigation. This analysis uses the values of the correlation coefficients between samples of pairs of twins $\mathrm{MZ}$ and $\mathrm{DZ}$ in the same formula $[2,35]$. Nevertheless, a weakness of the $h^{2}$ coefficient is that values less than 0 or greater than 1 can be found, which may reflect errors due to the small sample size. Besides that, the $h^{2}$ do not provide estimates of the role of the common/specific environment; therefore, the derived heritability estimates may represent upper limits of the true values [7]. The development of model-fitting methods to analyze twin data has made it possible to estimate the strength of genetic and environmental contributions at calculable confidence intervals. With modelfitting approach, it is possible to determine the proportion of the total variation explained by the additive/dominant genes and common/specific environment $[2,20]$. Hence, model-fitting methods that allow statistically testing the quality of fit of various genetic and environmental models provide more accurate data from twin's studies.

\section{Clinical and research implications}

In orthodontic practice, it should be considered that each malocclusion occupies its own distinct space in the genetic/environmental scenario and, therefore, the purpose of the overall diagnosis approach is to determine the extent of genetic vs. environment influences on specific malocclusion traits. The difficulty is that it is rarely feasible to estimate the exact relative contribution of hereditary and environment in a given patient. The available evidence may contribute, with a low certainty level, to the identification of the genetic and environmental contribution in certain patterns of dentoalveolar malocclusions, which can contribute to the success of orthodontic treatment planning.

This systematic review suggests that some occlusal characteristics such as overjet, overbite, and molar relationship have a high environmental component. In addition, an altered morphology in one of the dental arches will not necessarily lead to an alteration in the opposite arch. Despite the possible predetermined skeletal component, with the early removal of the environmental etiological factor, it may be possible to influence the dentoalveolar morphology within certain parameters. In such cases, interceptive orthodontic treatment may allow occlusion to develop according to its fundamental genetic profile, with reduced disturbance due to environmental factors, habits, and non-physiological functional variables [36, 37]. Early preventive approaches may avert the complete establishment of malocclusions and offer later a less cumbersome orthodontic treatment or, in certain cases, a better final outcome.

Although the identification of occlusal characteristics with stronger genetical determination represents an important finding, this in itself does not fully explain their etiology. This is an open field for future research and further exploration of epigenetic variables in an attempt to explain the observed differences between MZ twin pairs $[2,5,38]$. The focus is on determining the extents of differences in overall genomic DNA methylation levels are likely to be available in a mid-term future. Once these DNA-level approaches are refined, our knowledge of the local-level epigenetic influences should improve, and we should be able to provide a more 
complete model of how genetics, epigenetics, and environment interact to influence the development of occlusion. Molecular therapy is being used in other fields of medicine [38, 39]; therefore, it is up to the orthodontic specialty to follow developments in molecular genetics. Identifying relevant genes mediating occlusal development would provide scientific evidence for a future monitoring and counseling patients predisposed to developing malocclusions.

\section{Limitations}

Results from studies in twin pairs are difficult to compare and inconsistency/similarity should be interpreted with caution due to differences in assessment of zygosity, sample size requirements, stage of maturity, and statistical methods used. In addition, it should be noted that heritability is population estimation and is less relevant to the individual patient. Therefore, it would be misleading to describe that the clinical traits with limited heritability and highly environmentally influenced are often more prone to prevent and treat at the individual level of patients.

$\mathrm{MZ}$ and DZ twins were included in the review population. The pairs of MZ twins share the same genes, while the pairs of DZ twins share only half of their genes on average. By assuming that both types of twins are sampled from the same gene pool and that similar environmental factors affect them, one can estimate the contributions related to genetics and environmental influences to the variation observed in different traits [40]. However, the ideal model should include a heterogeneous population of twins according to zygosity. Only one study [19] in this review included a heterogeneous sample of MZ twins. Owing to the included studies that did not present separate heritability estimates for some variables, this may directly reflect the impact of heredity on occlusal traits.

Another concern in many twin research has been the precision of zygosity determination [2]. Two studies [19, 21] included in this review did not report information about the zygosity test. Although comparisons of physical appearance and date of birth may provide a reasonably reliable means of determining zygosity, errors may occur and may influence subsequent analyzes.

\section{Conclusion}

Based on a low level of certainty, the available evidence reports that few oclusal traits are under a potentially more dominant heritability influence, namely, the dental arch morphology (width, length, and shape) and the alignment/spacing teeth ratio, mainly for the mandibular arch. Nevertheless, there is no scientific evidence so far of genetic variables modulating the presence of asymmetry in the maxilla or mandible arches.
It appears that the traits concerning to the occlusal relationship between the maxilla and the mandible, such as overjet, overbite, posterior crossbite, and sagittal molar relationship, seem to have the environmental component as the major determinant.

\section{Supplementary information}

Supplementary information accompanies this paper at https://doi.org/10. 1186/s40510-020-00330-8.

Additional file 1. Search strategy in the different databases.

\section{Abbreviations}

DZ: Dizygosity; GRADE: The Grading of Recommendations Assessment, Development and Evaluation; MZ: Monozygosity twins; PAR: Peer Assessment Rating; PRISMA: Preferred Reporting Items for Systematic Review and MetaAnalysis; Q-Genie: Quality of Genetic Association Studies checklist

\section{Acknowledgements}

Not applicable.

\section{Funding}

The authors thank the CAPES (Coordenação de Aperfeiçoamento de Pessoal de Nivel Superior) for supporting academic and professional development. The funder had no role in study design, data collection, and analysis or preparation of the manuscript.

Availability of data and materials

The datasets used and/or analyzed during the current study are available from the corresponding author on reasonable request.

Ethics approval and consent to participate

Not applicable.

Consent for publication

Not applicable.

\section{Competing interests}

None declared.

\section{Author details}

${ }^{1}$ Department of Pediatric Dentistry and Orthodontics, Universidade Federal dos Vales do Jequitinhonha e Mucuri, Diamantina, Minas Gerais, Brazil. ${ }^{2}$ Department of Orthodontics, University of Alberta, Edmonton, Alberta, Canada. ${ }^{3}$ Department of Orthodontics, Complutense University of Madrid, Madrid, Spain. ${ }^{4}$ Department of Orthodontics, Southwest Bahia State University, Jequié, Bahia, Brazil.

Received: 23 March 2020 Accepted: 19 July 2020

Published online: 31 August 2020

References

1. Visscher PM, Goddard ME. A general unified framework to assess the sampling variance of heritability estimates using pedigree or marker-based relationships. Genetics. 2015;199(1):223-32.

2. Townsend $G$, Hughes $T$, Luciano M, Bockmann M, Brook A. Genetic an environmental influences on human dental variation: A critical evaluation of studies involving twins. Archives of Oral Biology. 2009:54:S45-51.

3. Kang KW, Corey LA, Evans MM, Christian JC, Norton JA. Dominance and environmental variances: their effect on heritabilities estimated from twin data. Hum Hered. 1977;27(1):9-21.

4. Djordjevic J, Zhurov Al, Richmond S, Consortium V. Genetic and Environmental Contributions to Facial Morphological Variation: A 3D Population-Based Twin Study. PLoS One. 2016;11(9):e0162250.

5. Mossey PA. The heritability of malocclusion: part 2. The influence of genetics in malocclusion. Br J Orthod. 1999;26(3):195-203. 
6. Townsend GC, Corruccini RS, Richards LC, Brown T. Genetic and environmental determinants of dental occlusal variation in South Australian twins. Aust Orthod J. 1988;10(4):231-5.

7. Eguchi S, Townsend GC, Richards LC, Hughes T, Kasai K. Genetic contribution to dental arch size variation in Australian twins. Arch Oral Biol. 2004;49(12):1015-24.

8. Sreedevi G, Srinivas B, Reddy TP, Prasad CM, Kumar BS, Satish R. Heritability of thirty cephalometric parameters on monozygotic and dizygotic twins: twin study method. J Contemp Dent Pract. 2013;14(2):304-11.

9. Harris EF, Smith RJ. Occlusion and arch size in families. A principal components analysis. Angle Orthod. 1982;52(2):135-43.

10. Švalkauskienè V, Šmigelskas K, Šalomskienè L, et al. Heritability estimates of dental arch parameters in Lithuanian twins. Stomatologija. 2015;17(1):3-8.

11. King L, Harris EF, Tolley EA. Heritability of cephalometric and occlusal variables as assessed from siblings with overt malocclusions. Am J Orthod Dentofacial Orthop. 1993;104(2):121-31.

12. Lobb WK. Craniofacial morphology and occlusal variation in monozygous and dizygous twins. Angle Orthod. 1987;57(3):219-33.

13. Kawala B, Antoszewska J, Necka A. Genetics or environment? A twinmethod study of malocclusions. World J Orthod. 2007;8(4):405-10.

14. Moher D, Liberati A, Tetzlaff J, Altman DG, Group P. Preferred reporting items for systematic reviews and meta-analyses: the PRISMA statement. J Clin Epidemiol. 2009;62(10):1006-12.

15. Sohani ZN, Meyre D, de Souza RJ, et al. Assessing the quality of published genetic association studies in meta-analyses: the quality of genetic studies (Q-Genie) tool. BMC Genet. 2015;16:50.

16. Guyatt $\mathrm{GH}$, Oxman AD, Vist GE, et al. GRADE: an emerging consensus on rating quality of evidence and strength of recommendations. BMJ. 2008; 336(7650):924-6.

17. Corruccini RS, Yap Potter RH. Genetic analysis of occlusal variation in twins. American Journal of Orthodontics. 1980;78(2):140-54.

18. Christian JC, Kang KW, Norton JJ. Choice of an estimate of genetic variance from twin data. Am J Hum Genet. 1974;26(2):154-61.

19. Kučević E, Pavlović J, Poštić SD, Čutović T, Todić J. Analysis of occlusal characteristics of identical homozygous twins. Vojnosanitetski Pregled. 2017; 74(12):1128-33.

20. Sidlauskas M, Salomskiene L, Andriuskeviciute I, et al. Heritability of mandibular cephalometric variables in twins with completed craniofacial growth. Eur J Orthod. 2016;38(5):493-502.

21. Richards LC, Townsend GC, Brown T, Burgess VB. Dental arch morphology in South Australian twins. Archives of Oral Biology. 1990;35(12):983-9.

22. Boraas JC, Messer LB, Till MJ. A Genetic Contribution to Dental Caries, Occlusion, and Morphology as Demonstrated by Twins Reared Apart. Journal of Dental Research. 1988;67(9):1150-5.

23. Sharma K, Corruccini R. Genetic basis of dental occlusal variations in northwest Indian twins. European Journal of Orthodontics. 1986;8(2):91-7.

24. Potter RH, Corruccini RS, Green LJ. Variance of occlusion traits in twins. J Craniofac Genet Dev Biol. 1981;1(2):217-27.

25. Boyd A, Golding J, Macleod J, et al. Cohort Profile: the 'children of the 90s'-the index offspring of the Avon Longitudinal Study of Parents and Children. Int J Epidemiol. 2013;42(1):111-27.

26. Moss ML, Salentijn L. The capsular matrix. Am J Orthod. 1969;56(5):474-90.

27. Trask GM, Shapiro GG, Shapiro PA. The effects of perennial allergic rhinitis on dental and skeletal development: a comparison of sibling pairs. Am J Orthod Dentofacial Orthop. 1987;92(4):286-93.

28. Devor EJ, McGue M, Crawford MH, Lin PM. Transmissible and nontransmissible components of anthropometric variation in the Alexanderwohl Mennonites: I. Description and familial correlations. Am J Phys Anthropol. 1986;69(1):71-82.

29. van der Linden FP. Genetic and environmental factors in dentofacial morphology. Am J Orthod. 1966;52(8):576-83.

30. Góis EG, Ribeiro-Júnior HC, Vale MP, et al. Influence of nonnutritive sucking habits, breathing pattern and adenoid size on the development of malocclusion. Angle Orthod. 2008;78(4):647-54.

31. Katz CR, Rosenblatt A, Gondim PP. Nonnutritive sucking habits in Brazilian children: effects on deciduous dentition and relationship with facial morphology. Am J Orthod Dentofacial Orthop. 2004;126(1):53-7.

32. Marković M. Results of a genetic study of triplets with class III malocclusion. Zahn Mund Kieferheilkd Zentralbl. 1983;71(2):184-90.

33. Lundstrom A, McWilliam JS. A comparison of vertical and horizontal cephalometric variables with regard to heritability. Eur J Orthod. 1987;9(2):104-8.
34. Savoye I, Loos R, Carels C, Derom C, Vlietinck R. A genetic study of anteroposterior and vertical facial proportions using model-fitting. Angle Orthod. 1998;68(5):467-70.

35. Smith C. Concordance in twins: methods and interpretation. Am J Hum Genet. 1974;26(4):454-66.

36. Jolley CJ, Huang GJ, Greenlee GM, Spiekerman C, Kiyak HA, King GJ. Dental effects of interceptive orthodontic treatment in a Medicaid population: interim results from a randomized clinical trial. Am J Orthod Dentofacial Orthop. 2010;137(3):324-33.

37. Sunnak R, Johal A, Fleming PS. Is orthodontics prior to 11 years of age evidence-based? A systematic review and meta-analysis. Journal of Dentistry. 2015;43(5):477-86.

38. Castillo-Fernandez JE, Spector TD, Bell JT. Epigenetics of discordant monozygotic twins: implications for disease. Genome Med. 2014;6(7):60.

39. Malki K, Koritskaya E, Harris F, Bryson K, Herbster M, Tosto MG. Epigenetic differences in monozygotic twins discordant for major depressive disorder. Transl Psychiatry. 2016;6(6):e839.

40. Townsend G, Hughes T, Bockmann M, Smith R, Brook A. How studies of twins can inform our understanding of dental morphology. Front Oral Biol. 2009;13:136-41. https://doi.org/10.1159/000242406. Epub 2009 Sep 21.

\section{Publisher's Note}

Springer Nature remains neutral with regard to jurisdictional claims in published maps and institutional affiliations.

\section{Submit your manuscript to a SpringerOpen ${ }^{\circ}$ journal and benefit from:}

- Convenient online submission

Rigorous peer review

- Open access: articles freely available online

High visibility within the field

- Retaining the copyright to your article

Submit your next manuscript at $>$ springeropen.com 